\title{
Salinity Interchange between Sea-Water in Sand and Overflowing Fresh-Water at Low Tide.
}

By

D. M. Reid,

Department of Biology, Harrow School.

With 1 Figure in the Text.

THE following investigations were carried out in order to test the validity of the oft-recurring statement to the effect that "burrowing organisms live under fresh-water conditions during low tide in estuaries." This type of statement is usually made in respect to organisms which live in the sand or mud of estuaries in which, at low tide, a stream of fresh water passes over the ground in which the organisms are buried.

I have not been able to find any references to show that work has ever been done on this subject with the idea of determining the actual salinity of the water held by such sand. Therefore I carried out the following experiments to try to find if data could be procured which would be sufficiently consistent to lead to any conclusions.

\section{LOCALITY.}

For preliminary purposes the experiments were carried out in a bay known as Traigh Allt Chailgeag on the North Coast of Scotland about three miles east of the village of Durness. Traigh Allt Chailgeag seemed very suitable for these experiments because,

1. The distance from H.W.M. to L.W.M. is short (250 yards),

2. At low tide a stream of fresh water flows across the sand,

3. The sand-grains are of fairly even size and the texture of the whole ground is uniform,

4. The animal population of the sand is small, since the bay is open to the north and is exposed to continuous heavy surf,

5. The slope is gentle and uniform,

6. There is a good depth of sand.

Apparatus.

The apparatus consisted of a number of glass tubes, each of 15 c.c. capacity, fitted with rubber stoppers; and a long glass pipette marked 
off in inches and fitted with a rubber bulb of about 100 c.c. capacity. For use in sand of even texture the glass of this pipette need only be $5 \mathrm{~mm}$. in internal diameter and $1 \mathrm{~mm}$. in wall thickness. Further, it should be of uniform bore without constrictions or swellings.

\section{Procedure.}

Observations were made at a number of stations at measured distances along the course of the stream from H.W.M. to L.W.M. following the retreating tide. At each station samples were taken of 1 , the flowing water of the stream, and 2, 3 and 4, the water in the sand at a depth of 2,4 and 6 inches respectively.

The taking of the first sample from each station presented no difficulties. Samples 2, 3 and 4, which were from the sand beneath the flowing water, were procured by compressing the bulb and pushing the graduated end of the pipette into the sand to the required distance. The bulb was then allowed to expand slowly so that a quantity of sand and water was drawn up the tube. If the pipette be withdrawn and held vertically the sand will collect towards the open end, whence it can be ejected and the superincumbent water poured into a collecting tube and labelled.

At each station, before taking the samples, the depth and surface speed of the flowing water were measured. The speed was estimated by floating a thin slab of cork, $6 \mathrm{in} . \times 5 \mathrm{in}$. in area, on the surface of the water and measuring with a stop-watch the time taken to cover a known distance.

The depth of the water over the sand was measured with a thin glass ruler placed so that one of its edges was against the flow of the water.

The temperature of the atmosphere and of the flowing water was then taken.

The results at first sight appear to be somewhat erratic, but allowance must be made for the difficulties in sampling, as it is impossible to avoid slight admixture of water from higher layers when taking samples of those below.

As was to be expected, the flowing water was found to increase steadily in salinity from H.W.M. to L.W.M., although the final salinity was much less than might have been anticipated.

From the curves (Fig. 1) showing the salinity of the water in the sand at different depths there appears to be a possibility of dividing the area between H.W.M. and L.W.M. into three well-defined sections as regards the water in the sand, viz. :-

1. A section from H.W.M. down shore for a distance of 90 yards in which the salinity increases with great rapidity. 
2. A middle section of about 60 yards in which there is very little increase in salinity.

3. A final section of about 100 yards extending to L.W.M. in which the rate of increase of the salinity is comparable to that of the first section.

This would seem to indicate that towards H.W.M. and L.W.M. the salinity of the water, at least in the shallower layers of the sand, is subject to a certain amount of fluctuation with the tides, whereas in the middle portion there exists a condition of greater stability which may be explained in the following way. In each of the three sections a different set of conditions prevail. Section 1 is under fresh water for the greater portion of 12 hours ; Section 3 is under sea-water for the greater portion of 12 hours, while Section 2 is under fresh and sea-water alternately for approximately equal periods. Also, during neap tides Section 1 is never under sea-water, Section 3 is always under sea-water, but Section 2 is subject to both sea- and fresh water.

At most, however, the exchange in salinity between the overflowing water and the water in the sand must be extremely slow. For instance, although these readings were taken within a short period of a spring tide the salinity of the water in the sand at H.W.M. and L.W.M. at 6-inch depth was considerably different from that of the overlying water. The two areas over which the graphs are drawn is an attempt to express this in another way. Assuming that the slope of the beach is a straight line between high- and low-water mark, the time that any point between H.W.M. and L.W.M. is under fresh water or sea-water can be found approximately by comparing the relative lengths into which a vertical from that point is cut by the harmonic curve.* For example, if it be desired to determine the time during which a point 180 yards below H.W.M. is under fresh water, it is only necessary to take the vertical AXB. Then the proportions of sea and fresh water will be (since total time is $\mathrm{AXB}) \frac{\mathrm{AX}}{\mathrm{AXB}}$ and $\frac{\mathrm{XB}}{\mathrm{AXB}}$ or $\frac{210}{300}$ and $\frac{90}{300}$ (hours) respectively of the time between H.W. and L.W. of that tide.

\section{Depth and Speed of Flowing Water.}

The relation between these and the salinity of the water in the sand does not appear to be very clear. Certain factors, not met with in the case

* This simple harmonic curve was drawn in accordance with the formula $\mathrm{h}= \pm \frac{1}{2} \mathrm{r} \operatorname{Cos} \theta$ where $\mathrm{h}=$ height of tide above mean tide level.

$\mathrm{r}=$ range of tide.

$\theta$ is an angle $=\frac{\text { Interval from H. or L.W. }}{\text { Duration of rise or fall }} \times 180^{\circ}$. 


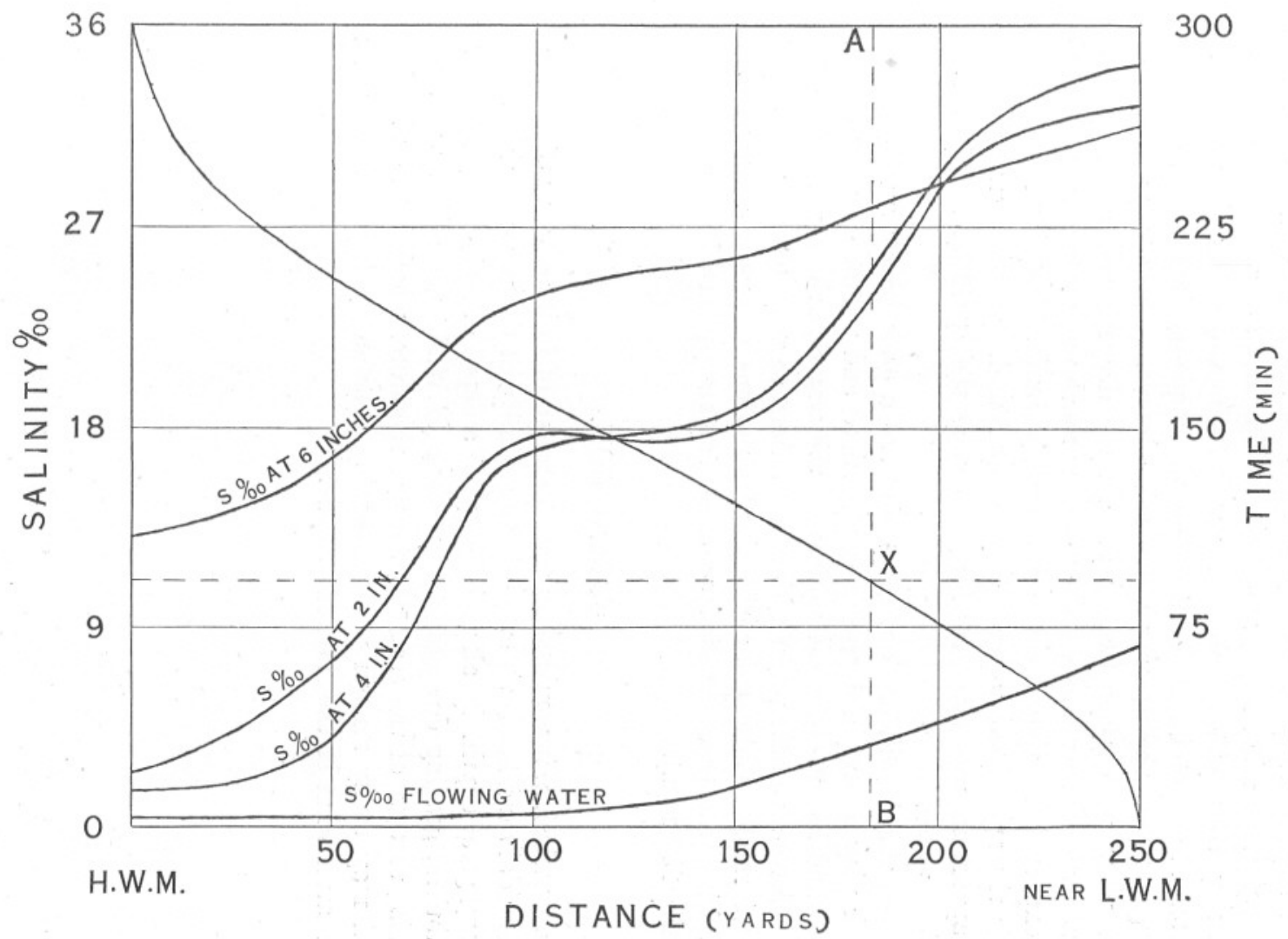

FIG, 1.-Curves representing the salinity of the water at various depths. These curves refer only to the times stated, they are not given as representing static nor minimum values. 
of streams which flow over a more coherent bed, also appear to disturb the relationship between depth and speed. When a stream of fresh water flows over loose sand of even texture ripple-marks are rapidly formed. These impede the progress of the water so that, in the first instance, although the surface layers may be travelling at a fair pace, eddy formation may cause other degrees of speed or direction of flow in the other layers. The second stage occurs when the ripple-marks form a bar great enough to force the stream to change its course. This change of course take place with great frequency. In the deeper pools, also, certain peculiarities are noticeable. The walls are much more vertical than might be expected, so that the through flow is limited to the surface layer while the rest of the water eddies slowly round. In these experiments no apparatus was available for estimating the speed of any but the surface water.

From this it will be seen that there are a great many small factors which play a part in determining the speed and volume of the flowing water and its effect on the salinity of the water in the sand. Some of these factors would appear to balance others, so that, for the present at least, it might be advisable to disregard variations in speed and depth, and, by taking an average, consider them as constant (speed $=1 \cdot 2$ feet per sec., depth $=250 \mathrm{~mm}$.).

\section{CONCLUSION.}

In conclusion, it would seem that a stream of fresh water passing over inter-tidal sand has little effect on the salinity of the water in that sand to any great depth. In all probability, at a depth of 10 inches (although, doubtless, grading down slightly towards H.W.M.) the salinity will be similar to that of the sea. Organisms, therefore, which burrow in the sand may easily remain under tolerably constant (salinity) conditions no matter what be the state of the tide. 